\title{
Optical glucose monitoring using vertical cavity surface emitting lasers (VCSELs)
}

\author{
Sahba Talebi Fard ${ }^{a}$ and Werner Hofmann $^{b}$ and Pouria Talebi Fard ${ }^{c}$ and Ezra Kwok ${ }^{d}$ and \\ Markus-Christian Amann ${ }^{b}$ and Lukas Chrostowski ${ }^{f}$ \\ ${ }^{a}$ IEEE Student Member, 2332 Main Mall,Vancouver, Canada; \\ ${ }^{b}$ Walter Schottky Institut, Technische Universitat Munchen, Am Coulombwall 3, D-85748 \\ Garching, Germany; \\ ${ }^{d}$ Director of Biomedical Engineering Program, 2360 East Mall, Vancouver, Canada; \\ ${ }^{f}$ IEEE Member, 2332 Main Mall, Vancouver, Canada;
}

\begin{abstract}
Diabetes Mellitus is a common chronic disease that has become a public health issue. Continuous glucose monitoring improves patient health by stabilizing the glucose levels. Optical methods are one of the painless and promising methods that can be used for blood glucose predictions. However, having accuracies lower than what is acceptable clinically has been a major concern. Using lasers along with multivariate techniques such as Partial Least Square (PLS) can improve glucose predictions. This research involves investigations for developing a novel optical system for accurate glucose predictions, which leads to the development of a small, low power, implantable optical sensor for diabetes patients.
\end{abstract}

\section{INTRODUCTION}

The burden of care for patients suffering from diabetes mellitus has been increasing. Continuous Glucose Monitoring (CGM) can effectively minimize the complications associated with diabetes. The current most common medical treatment for diabetes recommends four or more daily finger-prick glucose measurements, followed by an equivalent number of subcutaneous insulin injections or continuous insulin infusion. As a result of the open-loop nature of this method and poor patient compliance, glucose control and insulin delivery cannot be performed frequently enough. Several companies ${ }^{1,2}$ and research groups are striving to achieve continuous monitoring by developing long-term implantable chemical-based sensors either subcutaneous (e.g. under abdomen) ${ }^{3,4}$ or intravenous. $^{5,6}$ However, these enzyme-based sensors are restricted by their limited life spans, with drift and stability problems. One painless and promising technique is to use near-infrared spectroscopy, where an optical spectrum measurement is analyzed using multivariate techniques to determine glucose concentration. This method addresses the limitations of enzyme-based glucose sensors, since light is less susceptible to biofouling. A number of spectroscopic measurement techniques have been proposed by different researchers. ${ }^{7-15}$ These researchers either use transmission or reflection mode, ${ }^{15}$ with near-IR wavelengths spanning up to $10.5 \mu \mathrm{m} .{ }^{8}$ Multivariate techniques, specifically partial least square techniques, are used to analyze the optical spectrum measurements resulting from any of the above methods to determine glucose concentration. Non-invasive near infrared (NIR) glucose sensors are currently being developed by several companies. ${ }^{16,17}$ However, these non-invasive sensors have challenges due to interference, poor signal strength resulting in low signal-to-noise ratio, and calibration issues. The major concern with these sensors is achieving accuracies lower than what is acceptable clinically. These main challenges in accurate measurements would be significantly reduced if the optical sensor had close access to either interstitial fluid or preferably the blood plasma, so that the light did not have to interact with the several layers of tissue. To improve on the accuracy of the predictions, thermally tunable vertical cavity surface-emitting lasers (VCSELs) can be used to increase the signal-to-noise ratio in the spectrum. VCSELs are semiconductor lasers with small dimensions, low power consumption, and low cost. The goal of this research is to develop a small and low power implantable glucose sensor using VCSELs.

Combination and overtone molecular vibrations of $\mathrm{C}-\mathrm{H}$ and $\mathrm{O}-\mathrm{H}$ bonds of glucose molecule results in a broad absorption spectrum in two most promising wavelength region for glucose monitoring: the first-overtone (1560$1850 \mathrm{~nm})$ and the combination $(2.0-2.5 \mu \mathrm{m})$ bands. ${ }^{18-20}$ In these two promising wavelength regions, glucose has

Biosensing II, edited by Manijeh Razeghi, Hooman Mohseni, Proc. of SPIE Vol. 7397,

$739704 \cdot$ ? 2009 SPIE · CCC code: 0277-786X/09/\$18 · doi: 10.1117/12.828467 
numerous absorption bands and water has relatively lower absorption. The relative absorption is stronger in the combination band, which leads to about 20 times higher signal-to-noise ratio as compared to the first overtone band. ${ }^{21}$ Therefore, the combination band is a more desirable wavelength region for glucose spectroscopy.

VCSELs operate within a small spectrum with high power spectral density. To investigate the feasibility of using multi wavelength spectroscopy for glucose predictions using VCSELs, sets of white light experiments were performed on the first overtone wavelength band. As a result of these experiments, the optimum number of wavelength intervals can be determined. After investigating the possibility of using multi wavelength spectroscopy to predict glucose concentrations with white light, VCSEL-based experiments were performed on the combination band, which is a more desirable band for glucose spectroscopy. In these experiments, concentrations of glucose in various solutions were predicted using VCSELs as the source. Then, white-light experiments will be performed on both overtone and combination bands. Through these experiments, the most promising wavelength intervals can be determined, and VCSELs will be tuned to operate on the selected wavelength intervals.

\section{WHITE-LIGHT EXPERIMENTS}

This section includes results of investigation in the wavelength range of 1.0 to $1.7 \mu \mathrm{m}$. The wavelength region of interest in this experiment is the first overtone. The purpose of these experiments is to determine the minimum number of wavelength intervals required to effectively predict glucose concentration within an acceptable clinical accuracy using VCSEL-based sensors, and to assess the use of the partial least square (PLS) techniques for improvement given the absorption spectra at specific wavelength intervals. Most importantly, this section demonstrates that a segmented spectrum approach can be used and that the full spectrum is not necessary for glucose prediction.

\subsection{Apparatus for Glucose Spectroscopy Using White-Light}

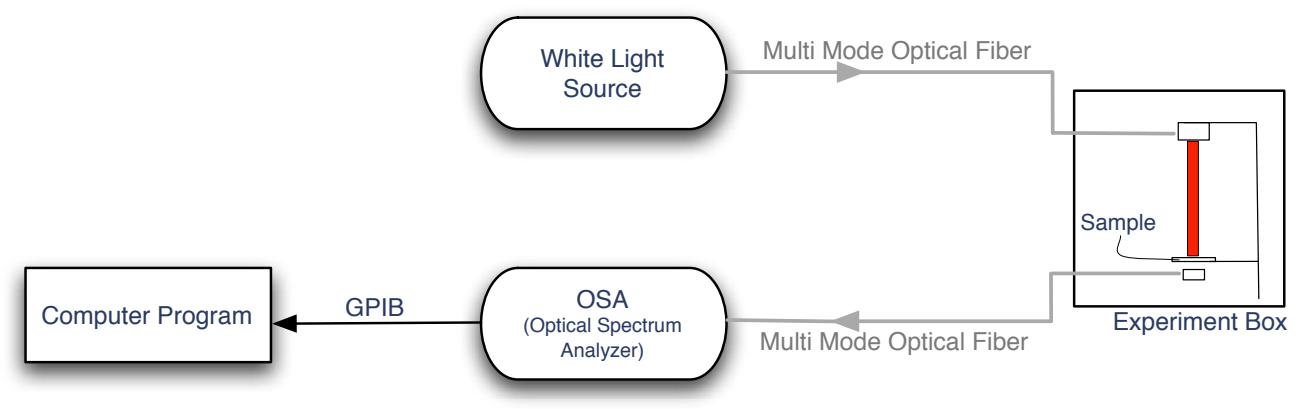

Figure 1. White-light experimental setup

The apparatus was set up such that the light from a white-light source goes through fiber collimators. It then passes through samples of aqueous glucose concentration. Another fiber collimator collects the light and transmits it to an optical spectrum analyzer (Figure 1). The sample holder consists of two slides of glass, with $1 \mathrm{~mm}$ thickness, separated by a $1 \mathrm{~mm}$ gap. Hence, the path length of light in the solution is $1 \mathrm{~mm}$. Aqueous solutions of glucose for various concentrations (0 to $1000 \mathrm{mM}$ ) have been used to construct absorption spectra for the wavelength $1.0 \mu \mathrm{m}$ to $1.7 \mu \mathrm{m}$. These higher glucose concentrations are used for preliminary investigations only. In the next VCSEL based experiments, more clinically relevant concentrations are used.

\subsection{Analysis and Results}

Two independent sets of 16 absorption spectra were measured using the set-up in Figure 1 . One set of 16 absorption spectra was used to calibrate the PLS model. The other set of 16 absorption spectra was used to validate the model and to estimate the accuracy of the predictions.

To find the intervals that can best predict the glucose concentration, the build-in function in the Matlab PLS toolbox, ${ }^{22}$ called interval PLS (iPLS), was used. First, the absorption spectra are preprocessed with Orthogonal 
Signal Correction (OSC) and mean centering. Then, iPLS searches for the wavelength interval or combination of wavelength intervals that can best predict the glucose concentration. Since each VCSEL can be thermally tuned over $7 \mathrm{~nm}$ of wavelength, the iPLS was designed to find five intervals of $7 \mathrm{~nm}$ width that show the best correlation with glucose concentrations. These chosen intervals are then used to build a PLS regression model. Figure 2 shows the placement of these intervals in the absorption spectrum.

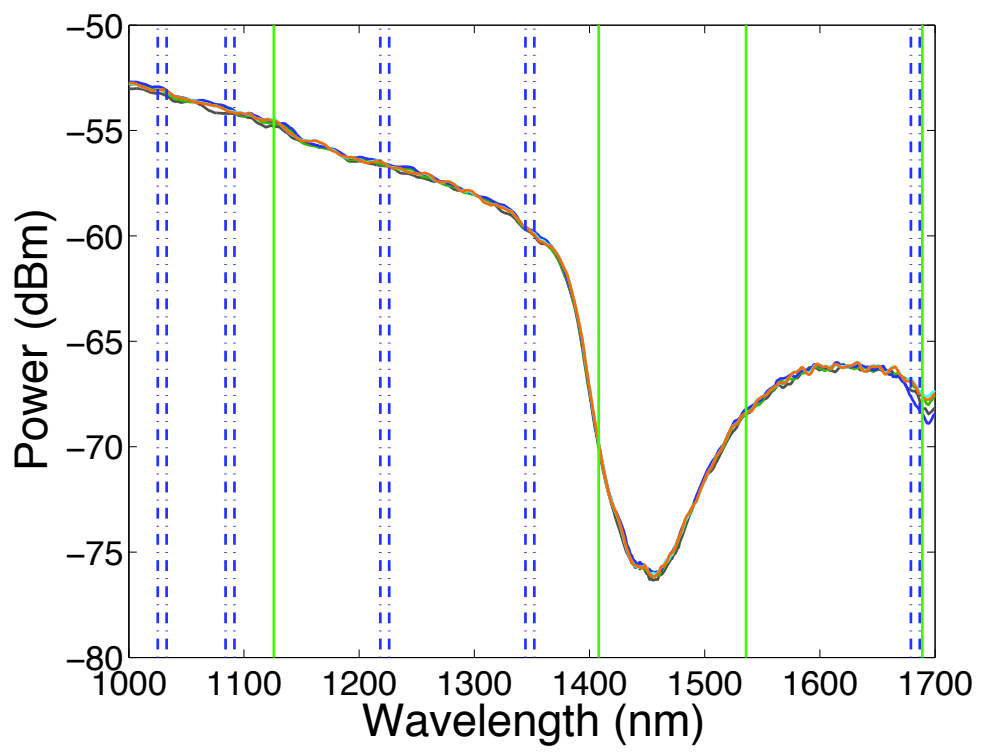

Figure 2. The 5 intervals of $7 \mathrm{~nm}$ width that best correlate with glucose concentration. The green solid vertical lines represent the glucose absorption peaks as given in the literature. The dashed lines show the chosen intervals, where each interval is $7 \mathrm{~nm}$ wide. The spectra have been preprocessed with Orthogonal Signal Correction (OSC) and mean centering. The wavelengths corresponding to these intervals are: 1025.2 to $1032.9 \mathrm{~nm} ; 1084$ to $1091.7 \mathrm{~nm} ; 1218.4$ to $1226.1 \mathrm{~nm}$; 1344.4 to $1352.1 \mathrm{~nm}$; and 1679 to $1686.7 \mathrm{~nm}$.

A PLS regression model is calibrated using five latent variables on these intervals preprocessed with OSC and mean centering. The result of glucose predictions using this model is presented in Figure 3, which shows that all the predictions are within the acceptable clinical accuracy. The prediction error is estimated to be $0.2837 \mathrm{mM}$ with this model.

\subsection{Discussion}

The results of these experiments show that multi-wavelength segmented spectroscopy is a feasible method for predicting glucose concentration. Therefore, small $(\sim 7 \mathrm{~nm})$ wavelength intervals may be used to predict glucose concentrations. In addition, analyzing the results of these experiments showed that increasing the number of wavelength intervals used for the glucose prediction model improves the accuracy of prediction. This accuracy improves with the number of wavelength intervals, and the improvement saturates at approximately 5 to 10 intervals. Figure 4 shows that increasing number of wavelength intervals improves accuracy.

\section{GLUCOSE SPECTROSCOPY USING VCSELS}

The feasibility of predicting glucose concentration using VCSELs is investigated in this section. These experiments use wavelength intervals from combination band which is a relatively more promising wavelength region for glucose spectroscopy. In addition, the results of predictions using a single VCSEL and two VCSELs are compared to validate the effect of an increased number of wavelength intervals on accuracy of predictions. 


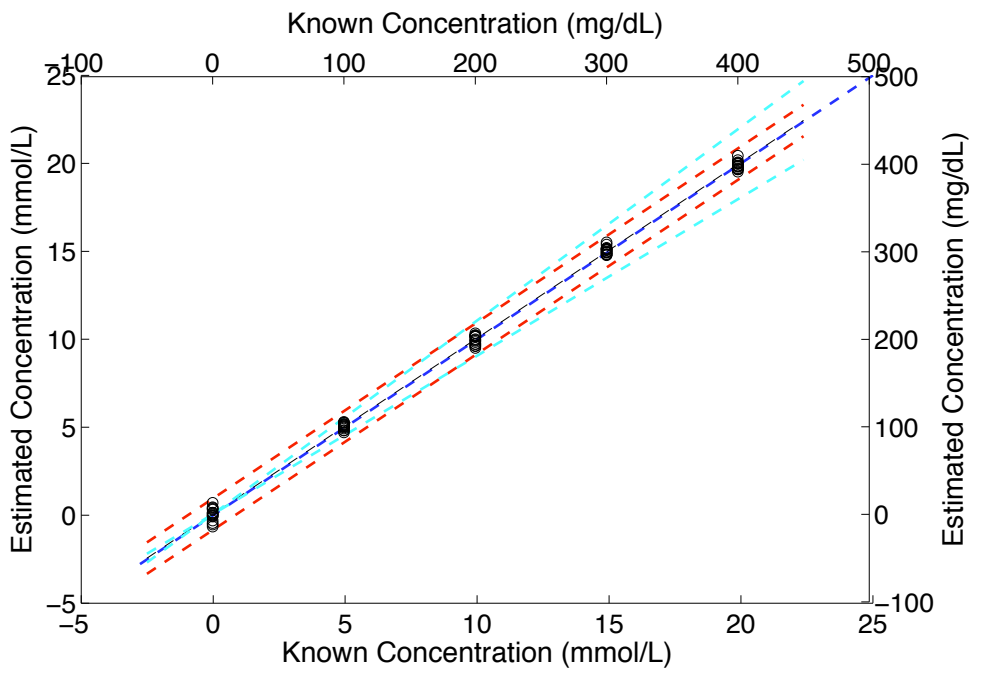

Figure 3. Glucose predictions using PLS with $5 \mathrm{LVs}$ and OSC with mean centering as the preprocessing on the set of five (5) intervals found by iPLS function. The plot represents predicted glucose concentration using the model vs. known concentration of those samples. The dashed red line defines the boundary for the predictions to be clinically acceptable $( \pm 1 \mathrm{mM})$; and the dashed green line shows the $10 \%$ boundary. The Root Mean Square Error of Prediction (RMSEP) value for this prediction is $5.1071 \mathrm{mg} / \mathrm{dL}$, equivalent to $0.2837 \mathrm{mM}$.

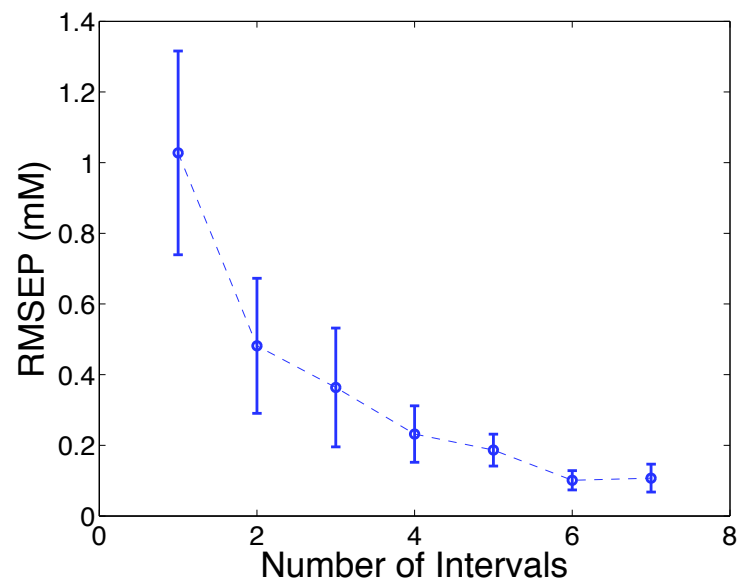

Figure 4. Error (RMSEP) vs. number of wavelength intervals used in model prediction. For example, using two wavelength segments of $7 \mathrm{~nm}$, as compared to using one, reduced error from about 1 to $0.5 \mathrm{mM}$. 


\subsection{Vertical Cavity Surface Emitting Lasers (VCSELs) for glucose Spectroscopy}

Semiconductor diode lasers are unique light sources with excellent spectral and beam properties. VCSELs are a type of semiconductor lasers that are attractive for implantable biomedical applications due to their low-cost, small size, array operation, low power consumption, and a circular beam pattern. For spectroscopy application, a range of wavelengths is required. This can be achieved through thermally tuning VCSELs by changing their supplied bias current which results in wavelength change. The range of wavelength change used for the VCSELs in this experiment is $4 \mathrm{~nm}$ at a center wavelength of $2.3 \mu \mathrm{m}$. Figure 5 shows optical spectra of the VCSEL for various bias currents. It shows that this VCSEL can achieve an emission wavelength of $2315 \mathrm{~nm}$, and it can be tuned over $5.6 \mathrm{~nm}$ if the driving current is changed from 10 to $28 \mathrm{~mA} .^{23}$
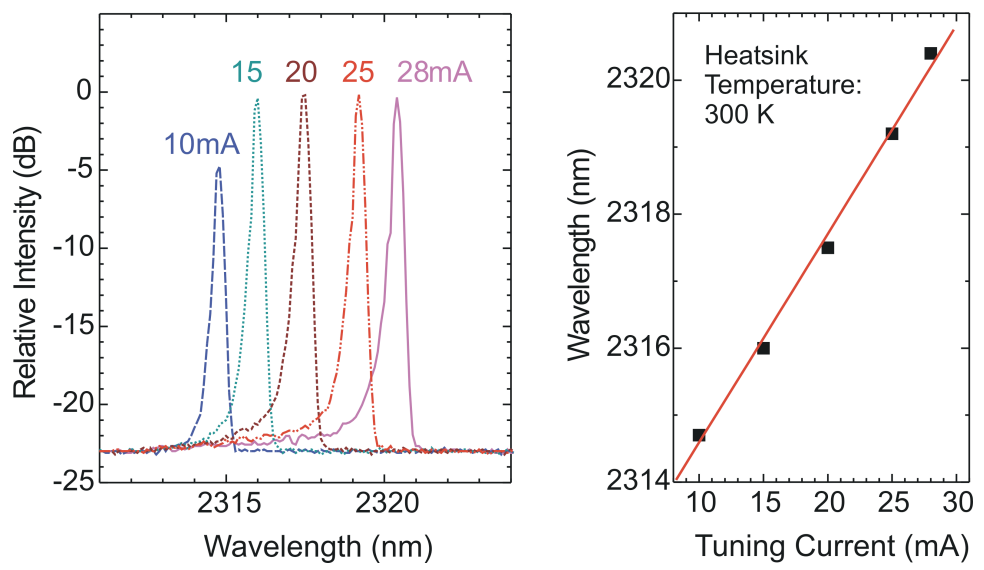

Figure 5. Current dependent optical output spectra of the $2.3 \mu \mathrm{m}$ LW-VCSEL, and wavelength shift with respect to bias current, at $300 \mathrm{~K}$, with the tuning slope of $0.3 \mathrm{~nm} / \mathrm{mA}$. The thermal tunability is $0.1 \mathrm{~nm} /{ }^{\circ} \mathrm{C}^{23}$

\subsection{Apparatus for Glucose Spectroscopy Using VCSELs}

Figure 6 shows the apparatus for the 2 VCSELs set-up. ${ }^{24}$

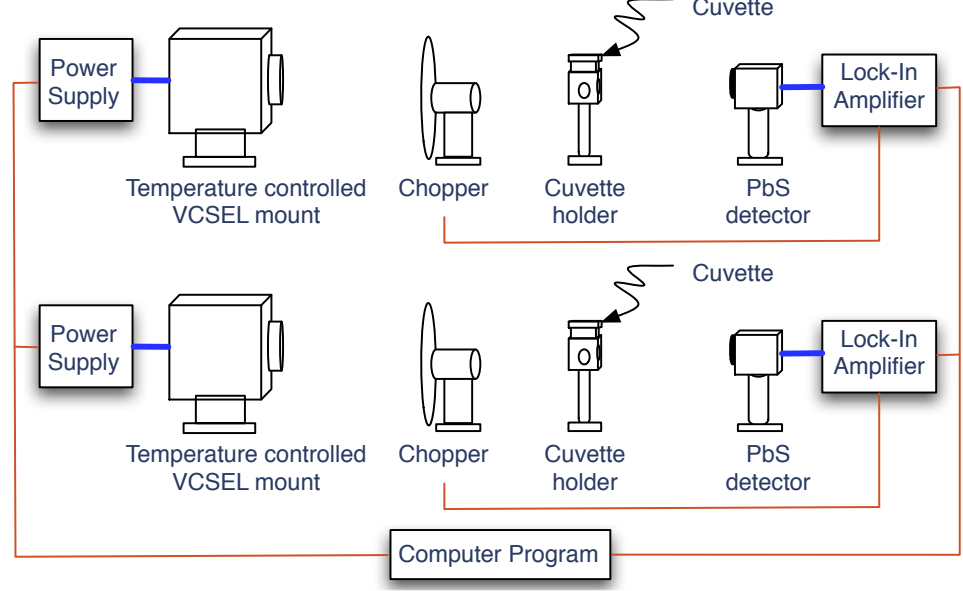

Figure 6. VCSEL-Based experimental setup. ${ }^{24}$

The $2.3 \mu \mathrm{m}$ VCSELs are current biased using a precision current source. VCSELs are temperature controlled at $25^{\circ} \mathrm{C}$ to improve the stability of their optical output. The optical output of the VCSEL is modulated at $250 \mathrm{~Hz}$ using a mechanical chopper. After passing through the sample, the beam is detected using a PbS pre-amplified detector. A lock-in amplifier is used to demodulate the signal to read the optical power and filter the data to remove noise components at other frequencies. 
Samples of glucose with various concentrations (about 0 to $25 \mathrm{mM}$ ) in three different solutions were prepared:

1. Aqueous solutions in distilled water

2. Physiological buffer solutions

3. Blood serum

These samples were stored in sealed plastic disposable cuvettes with a $2 \mathrm{~mm}$ path length.

\subsection{Analysis and Results}

Using the setup in Figure 6 nine transmission spectra for each glucose sample were measured using each VCSEL. Five of these transmission spectra are used to calibrate the prediction model and the remaining four are used to validate the model.

Figure 7 shows transmission spectra measured for samples of aqueous solutions of glucose. These measurements include the absorption spectral characteristics of the water, glucose, and cuvette, with the addition of noise. PLS techniques were used to calibrate the prediction model based on the changes that correlate well with the variations in glucose concentration.

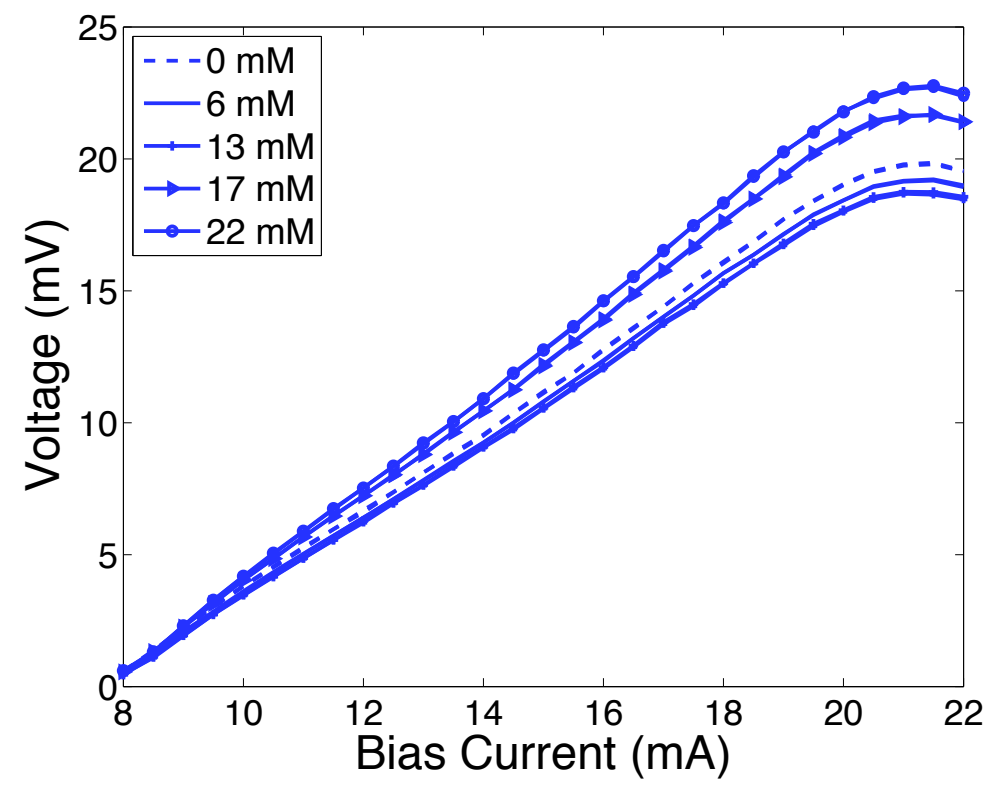

Figure 7. The measured transmitted optical power for various samples using a VCSEL. The VCSEL is biased with currents 8:0.5:22 $\mathrm{mA}^{24}$

To compare the accuracy of predictions and illustrate the significant improvements in predictions using two VCSELs rather than one VCSEL, PLS regression models have been calibrated using data collected with one single VCSEL as well as data collected with both VCSELs. These transmission spectra were preprocessed using OSC and mean centering.

Using transmission spectra from one single VCSEL, the PLS prediction model was constructed using two latent variables $(\mathrm{LVs})$. Figure 8a shows the glucose prediction results using this model. In the validation process, the RMSEP error was estimated to be $1.2 \mathrm{mM}$.

Furthermore, PLS prediction model was calibrated based on the transmission spectra measured with both VCSELs using four latent variables. The results of predictions were improved significantly as shown in Figure $8 \mathrm{~b}$, where the estimated RMSEP is $0.80 \mathrm{mM}$. 

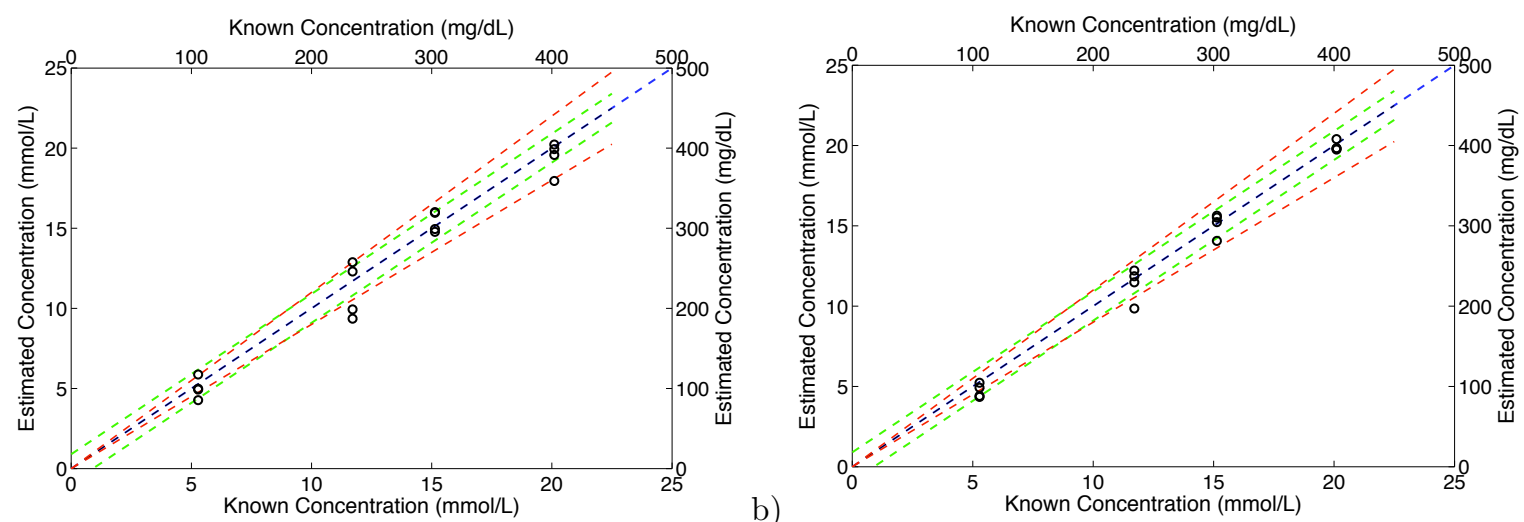

a)

b)

Figure 8. a) Glucose predictions with OSC and mean centered data from a single VCSEL for samples with lower concentration. PLS is used to calibrate regression model using 2 latent variables. The dashed green line defines the boundary for the predictions to be clinically acceptable $( \pm 1 \mathrm{mM})$; and the dashed red line shows the $10 \%$ boundary. The RMSEP error in the validation of this model determined to be $21.62 \mathrm{mg} / \mathrm{dL}$, equivalent to $1.2 \mathrm{mM} .{ }^{24} \mathrm{~b}$ ) Glucose predictions with the lower concentration data preprocessed using OSC and mean centering, from both VCSELs. PLS is used to calibrate regression model using 4 latent variables. The dashed green line defines the boundary for the predictions to be clinically acceptable $( \pm 1 \mathrm{mM})$; and the dashed red line shows the $10 \%$ boundary. The RMSEP error in the validation of this model is determined to be $14.4 \mathrm{mg} / \mathrm{dL}$, equivalent to $0.8 \mathrm{mM}^{24}$

Comparing the prediction results illustrated in Figures 8a and 8b indicates a significant improvement in accuracy of predictions using transmission spectra measure with two VCSELs rather than only one VCSEL.

A set of nine transmission spectra were measured from samples of glucose in physiological buffer using two different VCSELs. A PLS prediction model was then calibrated using five of these transmission spectra preprocessed with mean centering. The first prediction model used the spectra measured with a single VCSEL using two latent variables. Figure 9a shows the results of validation of this model with the remaining four spectra. The estimated RMSEP error for this model is $1.68 \mathrm{mM}$.
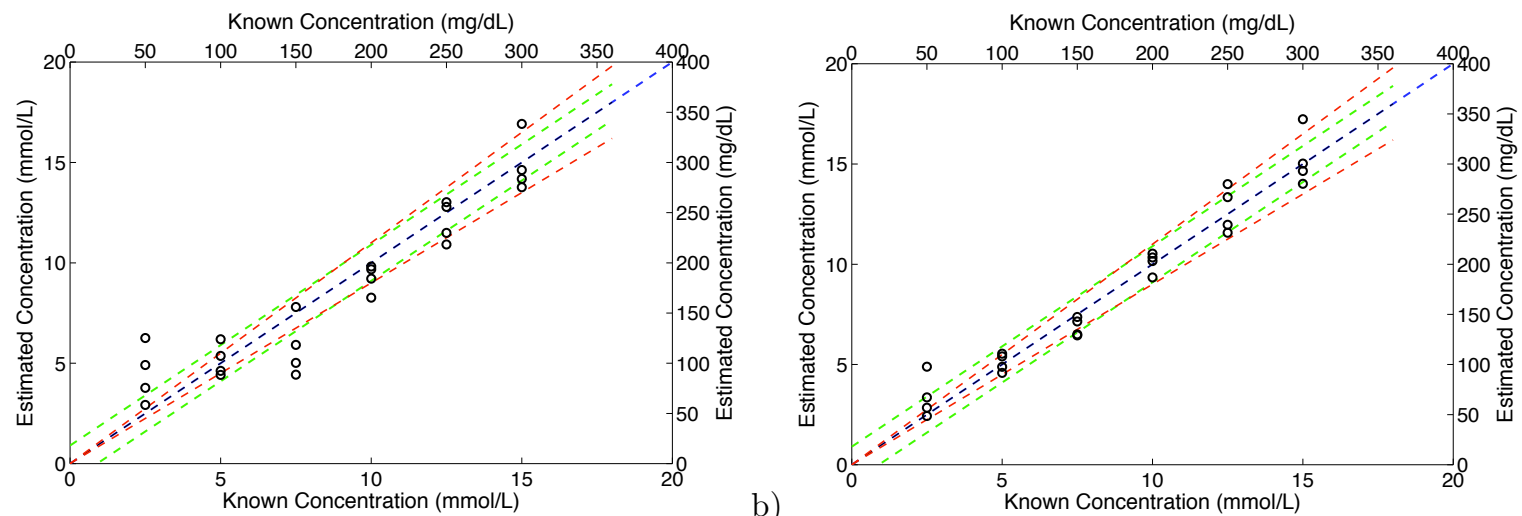

a)

b)

Figure 9. a) Glucose predictions based on the spectra from physiological buffer samples using one single VCSEL. PLS is used to calibrate regression model using 2 latent variables on the data preprocessed with mean centering. The dashed green line defines the boundary for the predictions to be clinically acceptable $( \pm 1 \mathrm{mM})$; and the dashed red line shows the $10 \%$ boundary. The RMSEP error in the validation of this model is determined to be $30.3 \mathrm{mg} / \mathrm{dL}$, equivalent to 1.68 $\mathrm{mM}$. b) Glucose predictions based on the spectra from physiological buffer samples using two VCSELs. PLS is used to calibrate regression model using 4 latent variables on the data preprocessed with mean centering. The dashed green line defines the boundary for the predictions to be clinically acceptable $( \pm 1 \mathrm{mM})$; and the dashed red line shows the $10 \%$ boundary. The RMSEP error in the validation of this model is determined to be $18.8 \mathrm{mg} / \mathrm{dL}$, equivalent to $1.04 \mathrm{mM}$.

The second prediction model was calibrated with the spectra measured with two VCSELs using four latent 
variables. Figure $9 \mathrm{~b}$ shows the results of validation of this model with the remaining four spectra. The estimated RMSEP error for this model is $1.04 \mathrm{mM}$.

Comparing the prediction results of these two models as illustrated in Figures 9a and 9b confirms that using spectra measured with two VCSELs significantly improves accuracy of predictions (RMSEP reduced from 1.68 to $1.04 \mathrm{mM})$.

\subsection{Discussion}

The results of the experiments in this section supported the feasibility of using VCSELs for predicting glucose concentration. To further illustrate the improved accuracy of predictions upon using two VCSELs, the estimated prediction errors using spectra of one VCSEL and two VCSELs were plotted in Figure 10 for various solutions of glucose (Aqueous, physiological buffer, and blood serum). Although the reduced estimated errors are not statistically significant enough, it is expected that the $95 \%$ confidence intervals will not overlap after more VCSELs are used and more experiments are conducted.

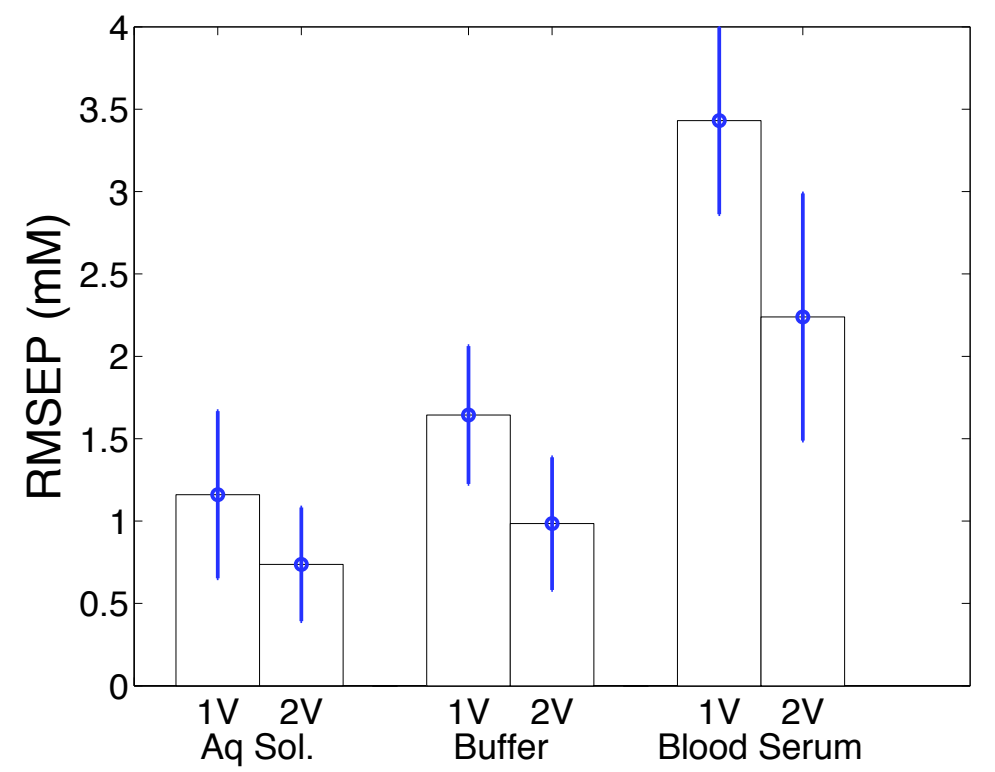

Figure 10. Average prediction error using absorption spectra collected with one VCSEL vs. two VCSELs in various solutions (aqueous, physiological buffer, and blood serum). 1V means absorption spectra from one VCSEL are used; and $2 \mathrm{~V}$ means absorption spectra of two VCSELs are used. ${ }^{24}$

\section{RESULTS WITH BLOOD SERUM USING VCSELS}

Figure 11 shows transmission spectra for various concentrations of glucose in blood serum.

A glucose prediction model was calibrated using the transmission spectra collected using two VCSELs. These transmission spectra were preprocessed using mean centering. Partial Least Square (PLS) technique with three latent variables (LVs) was used to calibrate this model using five of the transmission spectra. Figure 12a shows validation of this model with all nine absorption spectra, which resulted in RMSEP of $1.96 \mathrm{mM}$.

To further evaluate the PLS prediction models, an alternative approach to calibrating the model was taken. All the samples corresponding to one of the glucose concentrations $(11.1 \mathrm{mM})$ were removed from the calibration process but were included in validation. Figure $12 \mathrm{~b}$ shows the result of validating this model. The results of this analysis confirms that the model can predict the glucose concentration of the samples that did not exist in calibration set with a similar accuracy. 


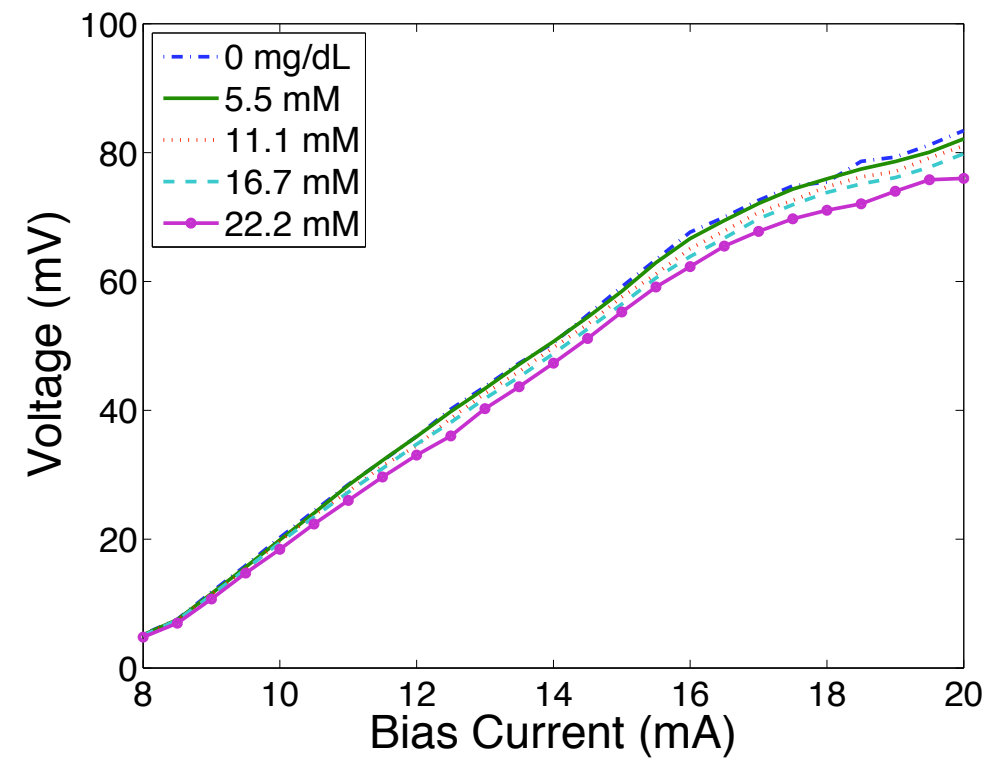

Figure 11. The measured transmitted optical power for various samples using a VCSEL. The VCSEL is biased with currents 8:0.5:20 mA. The samples are various concentrations of glucose in blood serum.

a)

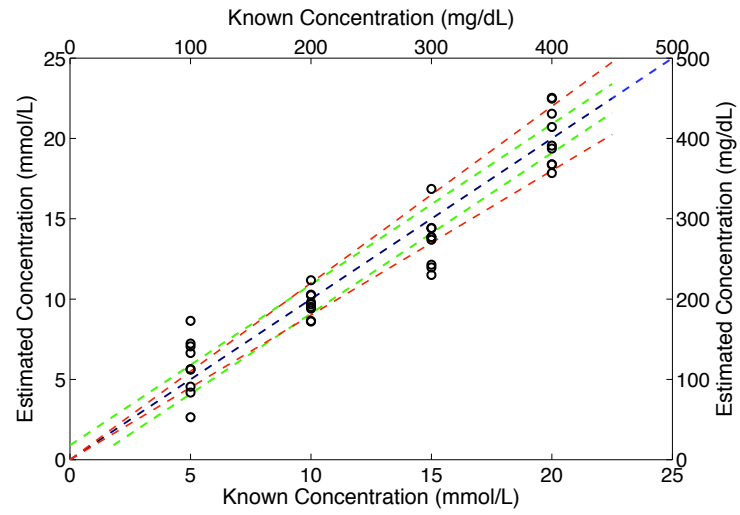

b)

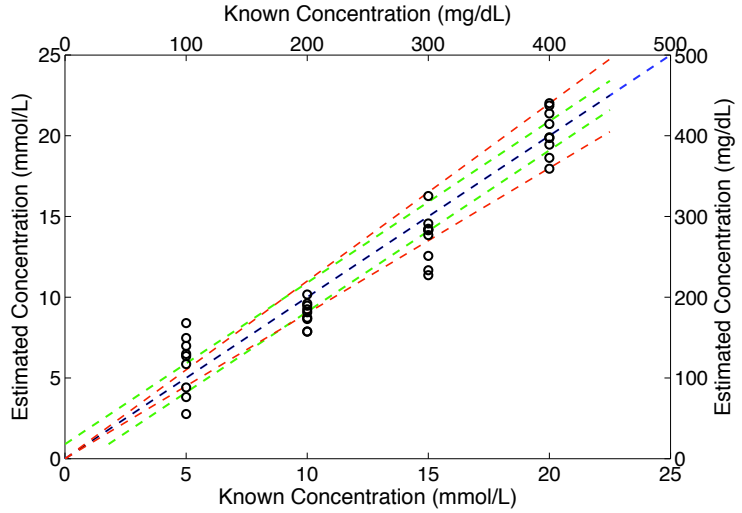

Figure 12. a) Results of glucose predictions using PLS regression model calibrated with five transmission spectra using three latent variables. The estimated RMSEP from the validation process is $1.96 \mathrm{mM}$. b) Results of glucose predictions using PLS regression model calibrated with all transmission spectra except one concentration (11.1 mM). The estimated RMSEP from the validation process is $2.2 \mathrm{mM}$. 


\section{CONCLUSION}

White light experiments are used to demonstrate that multi-wavelength segmented spectroscopy can provide sufficient glucose spectral information to predict the glucose concentration accurately. These experiments can further be used to specify the wavelength segments that are best correlated with glucose concentration as well as to determine the number of these segments required.

VCSEL-based experiments showed promising results in various solutions including blood serum. These results illustrated that VCSELs can be used for the purpose of glucose spectroscopy in a glucose sensor. In addition, Figure 10 confirmed that the accuracy of predictions is significantly improved if the model is calibrated using the transmission spectra from two VCSELs rather than using the spectra from a single VCSEL.

\section{ACKNOWLEDGEMENTS}

We acknowledge Canadian Diabetes Association (CDA), NSERC, and BC Innovation Council for supporting this research.

\section{REFERENCES}

[1] [Online]. Available: http://www.minimed.com/

[2] [Online]. Available: http://www.dexcom.com/

[3] S. S. Garg, Satish K. and and S. V. Edelman, "Improved glucose excursions using an implantable real-time continuous glucose sensor in adults with type 1 diabetes," Diabetes Care, vol. 27, 3, 2004.

[4] S. Updike, M. Shults, B. Gilligan, and R. Rhodes, "A subcutaneous glucose sensor with improved longevity, dynamic range, and stability of calibration," Diabetes Care, vol. 23, pp. 208-214, 2000.

[5] E. Renard, "Implantable closed-loop glucose-sensing and insulin delivery: the future for insulin pump therapy," Curr Opin Pharmacol, vol. 2, no. 6, pp. 708-716, 2002.

[6] J. Armour, J. Lucisano, B. McKean, and D. Gough, "Application of chronic intravascular glucose sensor in dogs," Diabetes, vol. 39, pp. 1519-1526, 1990.

[7] J. McNichols, Roger and L. Cote, Gerard, "Optical glucose sensing in biological fluids: An overview," Journal of Biomedical Optics, vol. 5, no. 1, pp. 5-16, January 2000.

[8] H. M. Heise, U. Damm, M. Bodenlenz, V. R. Kondepati, G. Kohler, and M. Ellmerer, "Bedside monitoring of subcutaneous interstitial glucose in healthy individuals using microdialysis and infrared spectrometry," Journal of Biomedical Optics, vol. 12, no. 2, p. 024004, 2007. [Online]. Available: http://link.aip.org/link/?JBO/12/024004/1

[9] J. Olesberg, L. Liu, V. Zee, and M. Arnold, "In vivo near-infrared spectroscopy of rat skin tissue with varying blood glucose levels," Analytical Chemistry, vol. 78, pp. 215-223, 2006. [Online]. Available: http://pubs3.acs.org/acs/journals/doilookup?in_doi=10.1021/ac051036i

[10] Y.-J. Kim, S. Hahn, and G. Yoon, "Determination of glucose in whole blood samples by mid-infrared spectroscopy," Appl. Opt., vol. 42, pp. 745-749, 2003. [Online]. Available: http://www.opticsinfobase.org/abstract.cfm?URI=ao-42-4-745

[11] K. Yamakoshi and Y. Yamakoshi, "Pulse glucometry: a new approach for noninvasive blood glucose measurement using instantaneous differential near-infrared spectrophotometry," Journal of Biomedical Optics, vol. 11, no. 5, p. 054028, 2006. [Online]. Available: http://link.aip.org/link/?JBO/11/054028/1

[12] Y.-J. Kim and G. Yoon, "Prediction of glucose in whole blood by near-infrared spectroscopy: Influence of wavelength region, preprocessing, and hemoglobin concentration," Journal of Biomedical Optics, vol. 11, no. 4, p. 041128, 2006. [Online]. Available: http://link.aip.org/link/?JBO/11/041128/1

[13] J. Olesberg, M. Arnold, C. Mermelstein, J. Schmitz, and J. Wagner, "Tunable laser diode system for noninvasive blood glucose measurements," Proc. SPIE, vol. 5702, pp. 23-29, 2005.

[14] S. F. Malin, T. L. Ruchti, T. B. Blank, S. N. Thennadil, and S. L. Monfre, "Noninvasive prediction of glucose by near-infrared diffuse reflectance spectroscopy," Clinical Chemistry, vol. 45, no. 9, pp. 1651-1658, 1999. 
[15] K. J. Jeon, I. D. Hwang, S. Hahn, and G. Yoon, "Comparison between transmittance and reflectance measurements in glucose determination using near infrared spectroscopy," Journal of Biomedical Optics, vol. 11, no. 1, p. 014022, 2006. [Online]. Available: http://link.aip.org/link/?JBO/11/014022/1

[16] [Online]. Available: http://www.inlightsolutions.com/

[17] [Online]. Available: http://www.sensysmedical.com/technology/index.html

[18] S. F.Malin, T. L. Ruchiti, T. B. Blank, S. U. Thennadil, and S. L. Monfre, "Noninvasive prediction of glucose by near-infrared diffuse reflectance spectroscopy," Clinical Chemistry, vol. 45, no. 9, pp. 1651-8, 1999.

[19] K. J. Jeon, I. D. Hwang, S. Hahn, and G. Yoon, "Comparison between transmittance and reflectance measurements in glucose determination using near infrared spectroscopy," Journal of Biomedical Optics, vol. 11, no. 1, pp. 014022-1: 014022-7, 2006.

[20] A. K. Amerov, J. Chen, G. W. Small, and M. A. Arnold, "Scattering and absorption effects in the determination of glucose in whole blood by near-infrared spectroscopy," Anal. Chem, no. 77, pp. 4587-4594, 2005.

[21] V. Saptari, K. Youcef-Toumi, and J. Zhang, "NIR measurements of glucose in synthetic biological solutions using high-throughput angle-tuned filter spectrometer," Optical Diagnostics and Sensing IV, vol. 5325, pp. $1-10,2004$.

[22] B. M. Wise, J. M. Shaver, N. B. Gallagher, W. Windig, R. Bro, and R. S. Koch, PLS Toolbox 4.0, Eigenvector Research Incorporated, 3905 West Eaglerock Drive, Wenatchee, WA 98801, 2006.

[23] S. T. Fard, W. Hofmann, P. T. Fard, G. Böhm, M. Ortsiefer, E. Kwok, M.-C. Amann, and L. Chrostowski, "Optical absorption glucose measurements using $2.3 \mu \mathrm{m}$ vertical cavity semiconductor lasers," IEEE Photonics Technology Letters, vol. 20, no. 11, pp. 930-932, 062008.

[24] S. T. Fard, E. Kwok, M.-C. Amann, and L. Chrostowski, "Chemometric approach for improving vcsel-based glucose predictions," IEEE transactions on Biomedical Engineering, submitted. 